\title{
Clinical study of 222 patients with pure motor stroke
}

\author{
A Arboix, I Padilla, J Massons, L García-Eroles, E Comes, C Targa
}

\begin{abstract}
The objective was to assess the frequency of pure motor stroke caused by different stroke subtypes and to compare demographic, clinical, neuroimaging, and outcome data of pure motor stroke with those of patients with other lacunar stroke as well as with those of patients with nonlacunar stroke.

Data from 2000 patients with acute stroke $(n=1761)$ or transient ischaemic attack $(n=239)$ admitted consecutively to the department of neurology of an acute care 350 bed teaching hospital were prospectively collected in the Sagrat Cor Hospital of Barcelona stroke registry over a 10 year period. For the purpose of the study 222 $(12.7 \%)$ patients with pure motor stroke were selected. The other study groups included $218(12.3 \%)$ patients with other lacunar strokes and 1321 (75\%) patients with non-lacunar stroke.
\end{abstract}

In relation to stroke subtype, lacunar infarcts were found in $189(85 \%)$ patients, whereas ischaemic lacunar syndromes not due to lacunar infarcts occurred in 23 (10.4\%) patients (atherothrombotic stroke in 12, cardioembolic stroke in seven, infarction of undetermined origin in three, and infarction of unusual aetiology in one) and haemorrhagic lacunar syndromes in $10(4.5 \%)$. Patients with pure motor stroke showed a better outcome than patients with non-lacunar stroke with a significantly lower number of complications and in hospital mortality rate, shorter duration of hospital stay, and a higher number of symptom free patients at hospital discharge. After multivariate analysis, hypertension, diabetes, obesity, hyperlipidaemia, non-sudden stroke onset, internal capsule involvement, and pons topography seemed to be independent factors of pure motor stroke in patients with acute stroke.

In conclusion, about one of every 10 patients with acute stroke had a pure motor stroke. Pure motor stroke was caused by a lacunar infarct in $85 \%$ of patients and by other stroke subtypes in $15 \%$. Several clinical features are more frequent in patients with pure motor stroke than in patients with non-lacunar stroke.

(F Neurol Neurosurg Psychiatry 2001;71:239-242)

Keywords: pure motor stroke; lacunar infarcts

Pure motor stroke is the most common and clinically best characterised of the lacunar syndromes. In this hospital based prospective study, we assessed the frequency of pure motor stroke caused by different stroke subtypes and the differential clinical features between pure motor stroke and other lacunar strokes. Demographic, clinical, neuroimaging, and outcome data of pure motor stroke and non-lacunar strokes were compared.

Patients and methods

Between 1986 and 1995, data from 2000 patients with acute stroke admitted consecutively to an acute care 350 bed teaching hospital in the city of Barcelona, Spain, were collected prospectively in a stroke registry. ${ }^{1}$ Transient ischaemic attacks were excluded. The study population consisted of 1761 patients with acute stroke. Subtypes of stroke were classified according to criteria of the Cerebrovascular Study Group of the Spanish Society of Neurology, ${ }^{2}$ which is similar to the National Institute of Neurological Disorders and Stroke classification. ${ }^{3}$ Definitions of cerebrovascular risk factors and lacunar syndrome (pure motor stroke, pure sensory stroke, sensorimotor stroke, ataxic hemiparesis, dysarthriaclumsy hand syndrome, and atypical lacunar syndromes) were those used in previous studies. ${ }^{45}$ Pure motor stroke was defined as a unilateral partial or complete paresis involving at least two of three areas (face, upper limb, or lower limb) of the body and no evidence of aphasia, apraxia, and agnosia, nor visual field defect, eye movement disturbance, ataxia, sensory loss, or evidence of bilateral weakness. Other lacunar syndromes were defined as producing pure sensory stroke, sensorimotor stroke, ataxic hemiparesis, dysarthria-clumsy hand, and atypical lacunar syndromes. Nonlacunar syndromes included all patients whose clinical picture did not conform with the preceding subgroups.

Lacunar syndromes diagnosed in 440 consecutive patients were collected. These included pure motor stroke in 222 patients, pure 
Table 1 Results of multivariate analysis: variables directly and inversely associated with pure motor stroke in the four logistic regression models

\begin{tabular}{|c|c|c|c|}
\hline Variables & $\beta$ & $S E(\beta)$ & OR $(95 \% C I)$ \\
\hline \multicolumn{4}{|c|}{ Demographic data and vascular risk factors: ${ }^{\star}$} \\
\hline Hypertension & 1.1942 & 0.1637 & $3.30(2.39-4.55)$ \\
\hline Obesity & 0.8052 & 0.3295 & $2.24(1.17-4.27)$ \\
\hline Diabetes & 0.4725 & 0.1798 & $1.60(1.13-2.28)$ \\
\hline Female sex & -0.3739 & 0.1581 & $0.69(0.50-0.94)$ \\
\hline Atrial fibrillation & -0.9531 & 0.2154 & $0.38(0.25-0.59)$ \\
\hline \multicolumn{4}{|c|}{ Demographic data, vascular risk factors and clinical features: $\dagger$} \\
\hline Hypertension & 1.2944 & 0.1690 & $3.65(2.62-5.08)$ \\
\hline Non-sudden stroke onset (h) & 0.6118 & 0.1571 & $1.84(1.14-2.36)$ \\
\hline Diabetes & 0.4988 & 0.1854 & $1.65(1.14-2.37)$ \\
\hline Hyperlipidaemia & 0.4134 & 0.2001 & $1.51(1.02-2.23)$ \\
\hline Speech disturbances & -0.6628 & 0.1617 & $0.51(0.37-0.71)$ \\
\hline Atrial fibrillation & -0.7863 & 0.2223 & $0.45(0.29-0.70)$ \\
\hline Headache & -0.9941 & 0.2651 & $0.37(0.22-0.62)$ \\
\hline Dizziness & -2.0106 & 0.7395 & $0.13(0.03-0.57)$ \\
\hline \multicolumn{4}{|c|}{ Demographic data, vascular risk factors, clinical features, and neuroimaging data: $\ddagger$} \\
\hline Internal capsule involvement & 2.7513 & 0.2360 & $15.66(9.86-24.87)$ \\
\hline Hypertension & 1.4479 & 0.1875 & $4.25(2.95-6.14)$ \\
\hline Diabetes & 0.6685 & 0.2047 & $1.95(1.31-2.91)$ \\
\hline Non-sudden stroke onset $(\mathrm{h})$ & 0.4397 & 0.1717 & $1.55(1.11-2.17)$ \\
\hline Speech disturbances & -0.7440 & 0.1796 & $0.47(0.33-0.67)$ \\
\hline Middle cerebral artery topography & -0.7915 & 0.2017 & $0.45(0.30-0.67)$ \\
\hline Headache & -1.1757 & 0.2788 & $0.31(0.18-0.53)$ \\
\hline Congestive heart failure & -1.5356 & 0.5766 & $0.21(0.07-0.67)$ \\
\hline Basal ganglia involvement & -1.9265 & 0.3140 & $0.14(0.08-0.27)$ \\
\hline Thalamic involvement & -2.4895 & 0.5340 & $0.08(0.03-0.24)$ \\
\hline \multicolumn{4}{|c|}{ Demographic data, vascular risk factors, clinical features, neuroimaging data, and outcome: $\S$} \\
\hline Internal capsule involvement & 2.6514 & 0.2398 & $14.17(8.86-22.68)$ \\
\hline Hypertension & 1.4829 & 0.1913 & $4.40(3.02-6.41)$ \\
\hline Diabetes & 0.7750 & 0.2108 & $2.17(1.44-3.28)$ \\
\hline Pons topography & 0.4370 & 0.3207 & $1.55(0.82-2.90)$ \\
\hline Middle cerebral artery involvement & -0.6475 & 0.2095 & $0.52(0.35-0.79)$ \\
\hline Speech disturbances & -0.7308 & 0.1834 & $0.48(0.34-0.69)$ \\
\hline Headache & -1.1022 & 0.2852 & $0.33(0.19-0.58)$ \\
\hline Urinary complications & -1.1058 & 0.3800 & $0.33(0.16-0.70)$ \\
\hline Rheumatic heart disease & -1.2722 & 0.7482 & $0.28(0.06-1.21)$ \\
\hline Congestive heart failure & -1.4847 & 0.5868 & $0.23(0.07-0.72)$ \\
\hline Cardiac events & -1.7466 & 0.7743 & $0.17(0.04-0.80)$ \\
\hline Neurological complications & -1.8952 & 0.5273 & $0.15(0.05-0.42)$ \\
\hline Thalamic involvement & -2.3771 & 0.5463 & $0.09(0.03-0.27)$ \\
\hline
\end{tabular}

$\star \beta=-2.6900, \mathrm{SE}(\beta)=0.4971$, goodness of fit $\chi^{2}=8.4581, \mathrm{df}=8, \mathrm{p}=0.3900$

$+\beta=-2.4153, \mathrm{SE}(\beta)=0.5368$, goodness of fit $\chi^{2}=12.2125, \mathrm{df}=8, \mathrm{p}=0.1420$

$\ddagger \beta=-2.1216, \mathrm{SE}(\beta)=0.5944$, goodness of fit $\chi^{2}=8.1408, \mathrm{df}=8, \mathrm{p}=0.4198$.

$\S \beta=-2.2323, \mathrm{SE}(\beta)=0.6102$, goodness of fit $\chi^{2}=9.6255, \mathrm{df}=8, \mathrm{p}=0.2923$

sensory stroke in 72 , sensorimotor stroke in 59 , ataxic hemiparesis in 17, dysarthria-clumsy hand in 25, and atypical lacunar syndrome in 45. Data from the 222 patients with pure motor stroke were selected. In all patients, diagnoses were established after diagnostic studies were performed and were consistent with definite diagnoses coded on the patient's discharge form. Atypical lacunar syndromes consisted of isolated dysarthria $(n=24)$, hemichoreahemiballismus $(n=5)$, unilateral paramedial thalamic infarction $(n=2)$, bilateral paramedial thalamic infarction $(n=3)$, pure motor hemiparesis with transient subcortical aphasia $(n=4)$, pure motor hemiparesis with transient internuclear ophthalmoplegia $(n=4)$, and isolated hemiataxia $(n=3)$. As defined in previous studies, ${ }^{14-6}$ pure motor stroke was attributed to a lacunar infarct, a cardioembolic infarction, an atherothrombotic infarction, a brain infarction of unusual aetiology, or a lacunar infarct of unknown cause.

All patients were admitted to hospital within 48 hours of stroke onset. Demographic characteristics, features of neurological examination, laboratory tests, chest radiography, and 12 lead electrocardiography were recorded. Brain CT was done within the first hospital week. Patients with negative results had a second CT during their stay in hospital or were studied by MRI ( $22 \%$ of patients). Other investigations included arterial digital subtraction angiography in $16 \%$ of patients, B mode echocardiography in $15 \%$, carotid duplex scan in $14 \%$, and lumbar puncture in $5 \%$. Outcome data included mortality in hospital, degree of clinical disability at discharge, and neurological complications, cardiac events, respiratory events, urinary events other than urinary tract infection, vascular complications, gastrointestinal complications, and infectious complications.

Student's $t$ test and the $\chi^{2}$ test (with Yate's correction when necessary) were used to compare features of patients with pure motor stroke with those of patients with other lacunar syndromes as well as with those of patients with non-lacunar stroke. Variables related to pure motor stroke in the univariate analysis plus age and sex were studied in multiple regression models based on demographic and vascular risk factors and clinical, neuroimaging, and outcome variables, in which pure motor stroke was the dependent variable.

\section{Results}

Of the 1761 patients with acute stroke, pure motor stroke was diagnosed in $222(12.7 \%)$. Other lacunar strokes were found in 218 patients and non-lacunar strokes in the remaining 1321. Lacunar infarcts were found in 189 $(85 \%)$ patients, whereas ischaemic lacunar syndromes not due to lacunar infarcts occurred in $23(10.4 \%)$ patients (atherothrombotic stroke in 12, cardioembolic stroke in seven, infarction of undetermined origin in three, and infarction of unusual aetiology in one) and haemorrhagic lacunar syndromes in 10 $(4.5 \%)$

In the 222 patients with pure motor stroke, there were 128 men and 94 women, with a mean (SD) age of 72.7 (10.3) years. Hypertension and diabetes were the most common vascular risk factors. Non-sudden onset of symptoms was found in $50 \%$ of patients. Speech disturbances occurred in 39\% of patients. Involvement of the internal capsule was the most frequent topography. The in hospital mortality was $0.5 \%(n=1)$. Symptom free at hospital discharge was recorded in 43 $(19.4 \%)$ patients. The mean duration of hospital stay was 13.7 (9.7) days.

There was a striking similarity between patients with pure motor stroke and patients with other lacunar strokes. However, limb weakness, involvement of the internal capsule, and middle cerebral artery topography were significantly more frequent in the pure motor stroke group, whereas sensory symptoms, thalamic involvement, and posterior cerebral artery topography were significantly more frequent in patients with other lacunar strokes. On the other hand, at univariate analysis, variables associated with pure motor stroke included male sex, hypertension, diabetes, obesity, smoking, hyperlipidaemia, non-sudden stroke onset, limb weakness, internal capsule involvement, pons involvement, and centrum 
ovale involvement. Variables significantly associated with non-lacunar stroke included rheumatic heart disease, atrial fibrillation, congestive heart failure, anticoagulation, sudden stroke onset, headache, dizziness, seizures, nausea, and vomiting, altered consciousness, sensory deficit, hemianopia, speech disturbances, basal ganglia involvement, medulla involvement, middle cerebral artery topography, and posterior cerebral artery topography. Patients with pure motor stroke showed a better outcome than patients with non-lacunar stroke with a significantly lower number of complications and in hospital mortality rate, shorter duration of hospital stay, and a higher number of symptom free patients at hospital discharge. After multivariate analysis, hypertension, diabetes, obesity, hyperlipidaemia, non-sudden stroke onset, involvement of the internal capsule, and pons topography seemed to be independent factors of pure motor stroke in patients with acute stroke (table 1).

\section{Discussion}

Pure motor stroke accounted for $12.7 \%$ of cases in the stroke registry and constituted $50 \%$ of lacunar syndromes. In agreement with previous studies, ${ }^{7-10}$ pure motor stroke is a reliable clinical test for diagnosing lacunar infarction. Accordingly, the concept of the lacunar hypothesis is clinically valid and useful. Although pathological heterogeneity in patients with pure motor stroke was found in $15 \%$ of our patients, the present results support the theory that pure motor stroke is commonly due to lacunar infarcts resulting from small vessel disease. ${ }^{11}$ However, roughly one in every seven patients with pure motor stroke may be associated with underlying non-lacunar ischaemic mechanisms, such as atherosclerosis, cardiac source of embolism, intracerebral haemorrhage, or spontaneous subdural haematoma that may influence management. Thus, because of implications for clinical management, particularly early thrombolytic therapy, ${ }^{12}$ it is mandatory to establish the aetiological diagnosis of pure motor stroke to classify correctly the stroke subtype.

Our findings showed a striking similarity in demographic data, vascular risk factors, and outcome between pure motor stroke and other lacunar strokes. However, there were important differences between pure motor stroke and non-lacunar strokes. In the multivariate analysis, hypertension, diabetes, hyperlipidaemia, and obesity were independent vascular risk factors significantly associated with pure motor stroke. Hypertension has been recognised as the single most important modifiable risk factor for acute stroke $^{13}$ with a maximal frequency of presentation in lacunar infarcts. ${ }^{14}{ }^{15}$ Diabetes is also a well known risk factor for lacunar infarction. Our results support the sufficient evidence to indicate that hypertension and diabetes have a direct effect on the occurrence of moderate to severe atherosclerotic stenosis of extracranial and intracranial arteries. ${ }^{16}$ Atherosclerosis and microangiopathy of coronary, peripheral, and cerebral arteries are often a complication of diabetes. Our results are in accord with those of a recent study ${ }^{17}$ in which history of diabetes and hyperlipidaemia were important risk factors for patients with lacunar strokes. Our findings indicate that obesity may be a more potent risk factor for pure motor stroke and lacunar infarcts than previously considered. The implication of lipohyalinosis (vasculopathy mainly responsible for small sized lacunar infarcts) in the association of obesity and lacunar stroke should be assessed in further clinicopathological studies. The fact that cardiac embolism is an unlikely mechanism for lacunar infarction is the reason for the inverse association between pure motor stroke and atrial fibrillation, rheumatic heart disease, and congestive heart failure.

Non-sudden stroke onset ${ }^{18}$ was an independent factor associated with pure motor stroke, whereas speech disturbances, headache, and dizziness were inversely associated with pure motor stroke. A non-sudden onset of neurological deficit was seen in $50 \%$ of pure motor stroke and in $32 \%$ of non-lacunar strokes. A slow onset characterises many lacunar infarcts by contrast with the sudden onset of most cases of major atheromatous or cardioembolic strokes. Headache occurred rarely and was inversely associated with pure motor stroke. It has been shown that headache is more frequent in haemorrhagic than in ischaemic stroke, and among patients with ischaemic stroke, the lacunar infarcts accounted for the lowest frequency of headache. ${ }^{19}{ }^{20}$ On the other hand, the lower frequency of speech disturbances in pure motor stroke may be due to the small lesion size and the more frequent topography at the subcortical level of the internal capsule or the pons and to a lower frequency of involvement of the territory of the middle cerebral artery compared with nonlacunar strokes.

The favourable outcome of patients compared with non-lacunar strokes was shown by a lower frequency of medical complications, in hospital mortality rate, and shorter duration of hospital stay. Patients with pure motor stroke showed significant spontaneous improvement with a higher frequency of symptom free at hospital discharge than patients with nonlacunar stroke.

We thank Drs M Balcells and M Oliveres for valuable participation in the study and Marta Pulido for editing the manuscript.

1 Arboix A, Vericat MC, Pujades R, et al. Cardioembolic infarction in the Sagrat Cor-Alianza Hospital of Barcelona Stroke Registry. Acta Neurol Scand 1997;96:407-12.

2 Arboix A, Alvarez-Sabín J, Soler L, for the Cerebrovascular Study Group of the Spanish Society of Neurology. Nomenclatura de las enfermedades vasculares cerebrales. Neurologia 1998; (suppl 1):1-10

3 Special Report from the National Institute of Neurological Disorders and Stroke. Classification of cerebrovascular diseases. III. Stroke 1990;21:637-76.

4 Arboix A, Martí-Vilalta JL. Lacunar syndromes not due to lacunar infarcts. Cerebrovasc Dis 1992;2:287-92.

5 Arboix A, Martí-Vilalta JL, García JH. Clinical study of 227 patients with lacunar infarcts. Stroke 1990;21:842-7.

6 Arboix A, García-Eroles L, Massons J, et al. Lacunar infarcts in patients aged 85 years and older. Acta Neurol Scand 2000;101:25-9.

7 Mohr JP, Caplan LR, Melski JW, et al. The Harvard Cooperative Stroke Registry: a prospective registry. Neurology

8 Richter RW, Brust JCM, Bruun B, et al. Frequency and course of pure motor hemiparesis: a clinical study. Stroke 1977;8:58-60 
9 Norrving B, Staaf G. Pure motor stroke from presumed lacunar infarct. Incidence, risk factors and initial course. Cerebrovasc Dis 1991;1:203-9.

10 Melo TP, Bogousslavsky J, van Melle G, et al. Pure motor stroke: a reappraisal. Neurology 1992;42:789-98.

11 Libman RB, Sacco RL, Shi T, et al. Neurologic improvement in pure motor hemiparesis: implications for clinical trials. Neurology 1992;42:1713-16.

12 Toni D, Iweins F, von Kummer R, et al. Identification of lacunar infarcts before thrombolysis in the ECASS I study. Neurology 2000;54:684-8.

13 Sacco RL, Benjamin EJ, Broderick JP, et al. Risk factors. Stroke 1997;28:1507-17.

14 You R, McNeil JJ, O’Malley HM, et al. Risk factors for lacunar infarction syndromes. Neurology 1995;45:1483-7.

15 Feigin VL, Wiebers DO, Nlkitin YP, et al. Risk factor for ischemic stroke in a Russian community. A populationbased case-control study. Stroke 1998;29:34-9.
16 Whisnant JP, Wiebers DO, O'Fallon WM, et al. A population-based model of risk factors for ischemic stroke. Rochester, Minnesota. Neurology 1996;47:1420-8.

17 Inzitari D, Eliasziw M, Sharpe BL, et al. Risk factors and outcome of patients with carotid artery stenosis presenting with lacunar stroke. North American Symptomatic Carotid Endarterectomy Trial Group. Neurology 2000;54:660-6.

18 Landi G, Cella E, Boccardi E, et al. Lacunar versus non-lacunar infarcts: pathogenetic and prognostic differences. F Neurol Neurosurg Psychiatry 1992;55:441-5.

19 Kumral E, Bogousslavsky J, van Melle G, et al. Headache at stroke onset: the Lausanne Stroke Registry. $f$ Neurol Neurosurg Psychiatry 1995;58:490-2.

20 Melo TP, Pinto AN, Ferro JM. Headache in intracerebral haematomas. Neurology 1996;47:494-500.

\section{1st Asia Pacific Forum on Quality Improvement in Health Care}

\section{Three day conference}

\section{Wednesday 19 to Friday 21 September 2001 Sydney, Australia}

We are delighted to announce this forthcoming conference in Sydney. Authors are invited to submit papers (call for papers closes on Friday 6 April), and delegate enquiries are welcome.

The themes of the Forum are:

- Improving patient safety

- Leadership for improvement

- Consumers driving change

- Building capacity for change: measurement, education and human resources

- The context: incentives and barriers for change

- Improving health systems

- The evidence and scientific basis for quality improvement.

Presented to you by the BMJ Publishing Group (London, UK) and Institute for Healthcare Improvement (Boston, USA), with the support of the the Commonweatlh Department of Health and Aged Care (Australia), Safety and Quality Council (Australia), NSW Health (Australia) and Ministry of Health (New Zealand).

For more information contact: quality@bma.org.uk or fax +44 (0) 73836869 